\title{
Lifting-Line Predictions for Induced Drag and Lift in Ground Effect
}

\author{
W. F. Phillips* \\ Utah State University, Logan, Utah 84322-4130 \\ and \\ D. F. Hunsaker \\ Scaled Composites, Mojave, CA 93501-1663
}

\begin{abstract}
Closed-form relations are presented for estimating ratios of the induced-drag and lift coefficients acting on a wing in ground effect to those acting on the same wing outside the influence of ground effect. The closed-form relations for these ground-effect influence ratios were developed by correlating results obtained from numerical solutions to Prandtl's liftingline theory. Results show that these influence ratios are not unique functions of the ratio of wing height to wingspan, as is sometimes suggested in the literature. These ground-effect influence ratios also depend on the wing planform, aspect ratio, and lift coefficient.
\end{abstract}

\section{Nomenclature}

$b \quad=$ wingspan

$C_{D i} \quad=$ wing induced-drag coefficient

$C_{L} \quad=$ wing lift coefficient

$h \quad=$ height of the wing above the ground

$R_{A} \quad=$ wing aspect ratio

$R_{T} \quad=$ wing taper ratio

$V_{y} \quad=y$-component of induced velocity

$V_{\infty} \quad=$ freestream airspeed

$x, y, z=$ streamwise, upward normal, and spanwise coordinates relative to the quarter chord midspan

$\bar{x}, \bar{y}, \bar{z}=x, y, z$ coordinates nondimensionalized relative to the wing semispan

$\alpha \quad=$ aerodynamic angle of attack

\footnotetext{
* Professor, Mechanical and Aerospace Engineering Department, 4130 Old Main Hill. Senior Member AIAA.

${ }^{\dagger}$ Design Engineer, 1624 Flight Line. Member AIAA.
} 
$\beta \quad=$ empirical correction coefficient, Eq. (7)

$\beta_{D}, \beta_{L}=$ high-lift correction coefficients, Eqs. (17) and (19)

$\delta_{D}, \delta_{L}=$ tapered-wing correction coefficients, Eqs. (13) and (15)

\section{Introduction}

Ground effect reduces the induced drag acting on a lifting wing. For wings of arbitrary planform with no geometric or aerodynamic twist, the induced-drag coefficient, $C_{D i}$, is proportional to the lift coefficient squared, $C_{L}^{2}$. Hence, a common measure of the influence of ground effect is the ratio of the induced-drag coefficient to the lift coefficient squared evaluated with the wing at some height $h$ above the ground, $\left(C_{D i} / C_{L}^{2}\right)_{h}$, divided by the same ratio evaluated with the wing outside the influence of ground effect, $\left(C_{D i} / C_{L}^{2}\right)_{\infty}$,

$$
\begin{aligned}
& \text { induced-drag ground- } \\
& \text { effect influence ratio }
\end{aligned} \equiv \frac{\left(C_{D i} / C_{L}^{2}\right)_{h}}{\left(C_{D i} / C_{L}^{2}\right)_{\infty}}
$$

Several different closed-form relations for this induced-drag ground-effect influence ratio have been recommended in aeronautics textbooks, which express the influence ratio as a function of a single dimensionless variable, $h / b$, where $h$ is the height of the wing above the ground and $b$ is the wingspan.

1. A relation of this form was recommended in 1975 by Hoerner and Borst [1],

$$
\frac{\left(C_{D i} / C_{L}^{2}\right)_{h}}{\left(C_{D i} / C_{L}^{2}\right)_{\infty}}=\frac{33(h / b)^{1.5}}{1+33(h / b)^{1.5}}
$$

More recently, Raymer [2] also recommends the use of this relation.

2. In the 1979 printing of the First Edition of his textbook McCormick [3] presented the relation,

$$
\frac{\left(C_{D i} / C_{L}^{2}\right)_{h}}{\left(C_{D i} / C_{L}^{2}\right)_{\infty}}=\frac{(16 h / b)^{2}}{1+(16 h / b)^{2}}
$$

This relation was repeated more recently by Anderson [4,5] and Phillips [6]. Over the range $0.05<h / b<1.0$, results obtained from Eq. (2) will significantly over predict the induced drag when compared with results obtained from Eq. (1). For example, near the point of maximum deviation, which occurs at about $h / b=0.1$, results predicted from Eq. (2) are nearly $41 \%$ above those predicted from Eq. (1).

3. The relation given in Eq. (2) is attributed to a typographical error, which was introduced by McCormick [3] and propagated by Anderson [4,5] and Phillips [6]. Somewhere between the first and the eighteenth printing of the First Edition of McCormick [3] the ground-effect relation given in Eq. (2) was corrected to read 


$$
\frac{\left(C_{D i} / C_{L}^{2}\right)_{h}}{\left(C_{D i} / C_{L}^{2}\right)_{\infty}}=\frac{[16 h /(\pi b)]^{2}}{1+[16 h /(\pi b)]^{2}}
$$

For $h / b<1.0$, Eq. (3) will significantly under predict the induced drag when compared with results obtained from Eq. (1). For example, at $h / b=0.1$, Eq. (3) predicts a result nearly $60 \%$ below that predicted by Eq. (1).

4. If the wing lift coefficient is small compared to the aspect ratio, a relation recommended by Torenbeek [7] in 1982 becomes a function of the single dimensionless variable, $h / b$,

$$
\frac{\left(C_{D i} / C_{L}^{2}\right)_{h}}{\left(C_{D i} / C_{L}^{2}\right)_{\infty}}=1-\exp \left[-2.48(2 h / b)^{0.768}\right]
$$

This agrees closely with the Hoerner and Borst [1] relation given by Eq. (1) over the range $h / b>0.08$. In this range Eq. (4) agrees with Eq. (1) to within about $\pm 6 \%$.

In the Second Edition of his textbook, McCormick [8] pointed out the inaccuracy of Eq. (3) for heights below a semispan, and he presented a new relation in graphical form, which was obtained from numerical computations for elliptic wings and covers the range $0.075<h / b<1.06$. The graphical relation presented by McCormick [8] agrees almost exactly with the Hoerner and Borst [1] relation given by Eq. (1) over the range $h / b>0.7$, and it agrees almost exactly with the Torenbeek [7] relation given by Eq. (4) over the range $h / b<0.3$. In fact, this graphical relation agrees with Eq. (4) to within less than $2 \%$ over the entire range, $0.075<h / b<1.06$.

It has been shown that the numerical lifting-line method of Phillips and Snyder [9] can be used to accurately predict the induced drag acting on a lifting wing. One convenient way to model an aircraft in ground effect using any potential flow algorithm is to replace the surface of the ground with an image of the aircraft, positioned and oriented as though it were reflected in the surface of the ground. Such a model is shown in Fig. 1. By design, the flow around this aircraft combined with its mirror image is symmetric across the plane of reflection. At any point on this plane of symmetry, the downwash generated by the aircraft will be exactly offset with upwash generated by its mirror image. Thus, there can be no net flow normal to the plane of reflection, which is accordingly a stream surface for the flow. This means that potential flow about the aircraft combined with its mirror image is identical to potential flow about the aircraft combined with a flat solid surface representing the ground. Since the two flows are identical, the associated aerodynamic forces will be identical as well. The model shown in Fig. 1 was used with the numerical lifting-line method of Phillips and Snyder [9] to predict the induced drag in ground effect for several untwisted wings of elliptic, rectangular, and tapered planforms. Results from these grid-resolved numerical lifting- 
line solutions at small aerodynamic angles of attack are presented in Fig. 2 compared with the relations presented by Hoerner and Borst [1], McCormick [3], and Torenbeek [7]. The numerical lifting-line results shown in Fig. 2 include aspect ratios of 4,8 , and 16 for each wing planform.

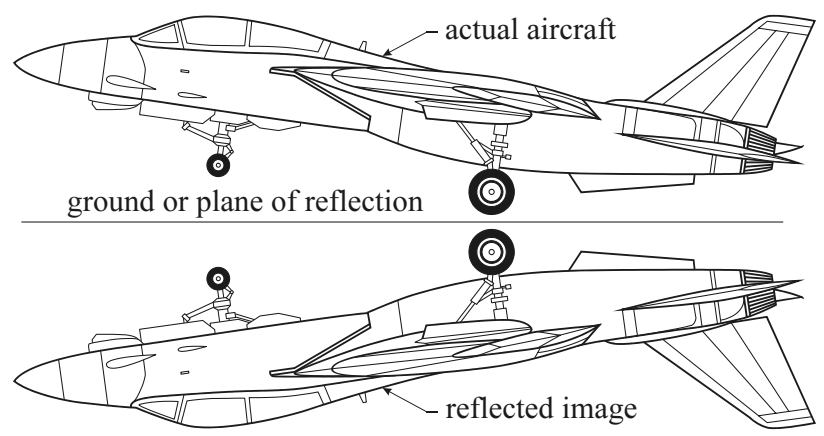

Fig. 1 Mirror image model used to simulate ground effect with potential flow algorithms.

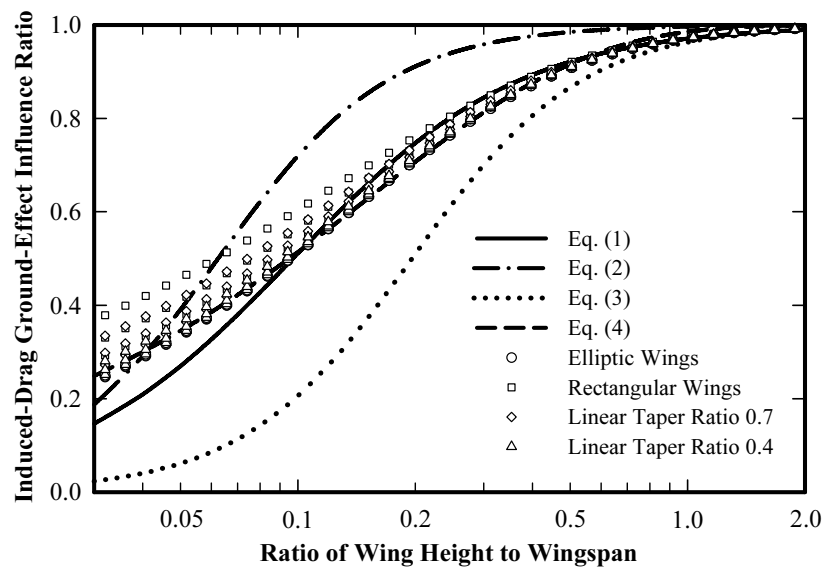

Fig. 2 Comparison of various relations for the induced-drag ground-effect influence ratio with results obtained from the numerical lifting-line method of Phillips and Snyder [9].

The results presented in Fig. 2 show that the relations of Hoerner and Borst [1] and Torenbeek [7] both agree closely with results obtained from the numerical lifting-line solutions for elliptic wings. The greatest discrepancy is in the range $h / b<0.07$, where Eq. (1) predicts induced drag that is somewhat below that predicted by Eq. (4) and the numerical lifting-line solutions for elliptic wings. In the range $h / b>0.07$, Eq. (4) agrees with the lifting-line results shown in Fig. 2 for elliptic wings to within 1.5\% and Eq. (1) agrees with these lifting-line results to within 7.5\%. For rectangular wings in the range $h / b<0.13$, both Eq. (1) and Eq. (4) predict induced drag that is somewhat below that predicted from the numerical lifting-line solutions. 
A slightly improved closed-form approximation for the mean of all numerical lifting-line solutions shown in Fig. 2 is obtained by using the relation of Torenbeek [7] with slightly modified coefficients,

$$
\frac{\left(C_{D i} / C_{L}^{2}\right)_{h}}{\left(C_{D i} / C_{L}^{2}\right)_{\infty}}=1-\exp \left[-4.01(h / b)^{0.717}\right]
$$

A comparison between Eq. (5) and the numerical lifting-line solutions is shown in Fig. 3.

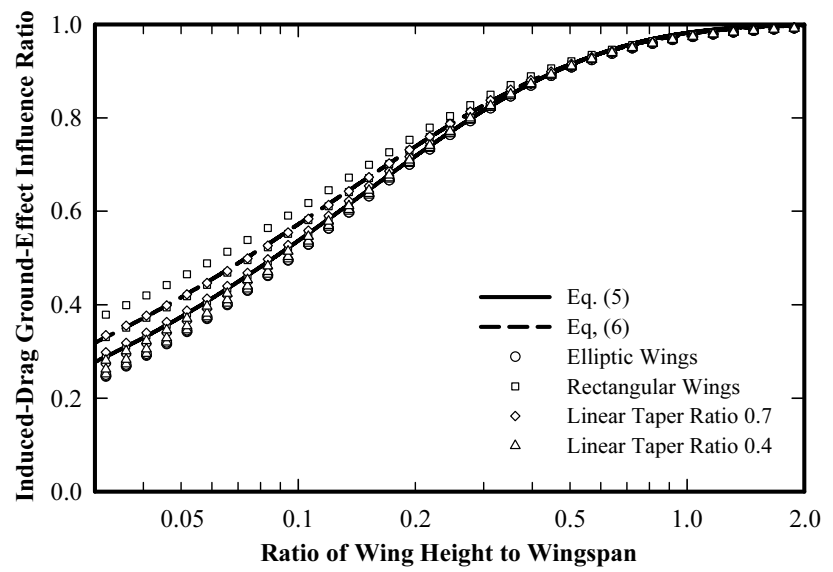

Fig. 3 Comparison of Eqs. (5) and (6) with results obtained from numerical lifting-line solutions.

The apparent scatter in the lifting-line solutions, which are shown in Figs. 2 and 3, results from the fact that the induced-drag ground-effect influence ratio is not a unique function of the single dimensionless variable, $h / b$. This influence ratio is also a weak function of wing planform and an even weaker function of wing aspect ratio. When $h / b$ is less than about 0.4 , the induced drag predicted from Eq. (5) for rectangular and slightly tapered wings is somewhat low, and that predicted for elliptic wings and wings with linear taper ratios near 0.4 is slightly high. A similar but more conservative relation is provided by fitting only the lifting-line solutions obtained for rectangular wings,

$$
\frac{\left(C_{D i} / C_{L}^{2}\right)_{h}}{\left(C_{D i} / C_{L}^{2}\right)_{\infty}}=1-\exp \left[-3.88(h / b)^{0.660}\right]
$$

This closed-form approximation is also shown in Fig. 3.

Torenbeek [7] also recognized that the induced-drag ground-effect influence ratio is not a unique function of the single dimensionless variable, $h / b$. To correct for this fact, Torenbeek [7] suggested a minor correction to Eq. (4), which for small values of $h / b$ predicts a slight increase in the influence ratio over that predicted by Eq. (4). 
When this correction is included, the Torenbeek [7] relation becomes a function of the wing lift coefficient, $C_{L}$, and aspect ratio, $R_{A}$, as well as the dimensionless height, $h / b$,

$$
\begin{array}{r}
\frac{\left(C_{D i} / C_{L}^{2}\right)_{h}}{\left(C_{D i} / C_{L}^{2}\right)_{\infty}}=\frac{1-\exp \left[-2.48(2 h / b)^{0.768}\right]}{1-\beta C_{L} /\left(4 \pi R_{A} h / b\right)}, \\
\text { where } \beta=\sqrt{1+(2 h / b)^{2}}-2 h / b
\end{array}
$$

Typical results predicted from Eq. (7) are compared with the small-angle numerical lifting-line solutions in Fig. 4. Equation (7) does not give reasonable results for very low values of $h / b$, because the relation contains a singularity in this region. For an aspect ratio of 6 and a lift coefficient of 0.5 , the singularity occurs at about $h / b=0.00655$. For an aspect ratio of 4 and a lift coefficient of 1.4 , the singularity occurs near $h / b=0.02642$.

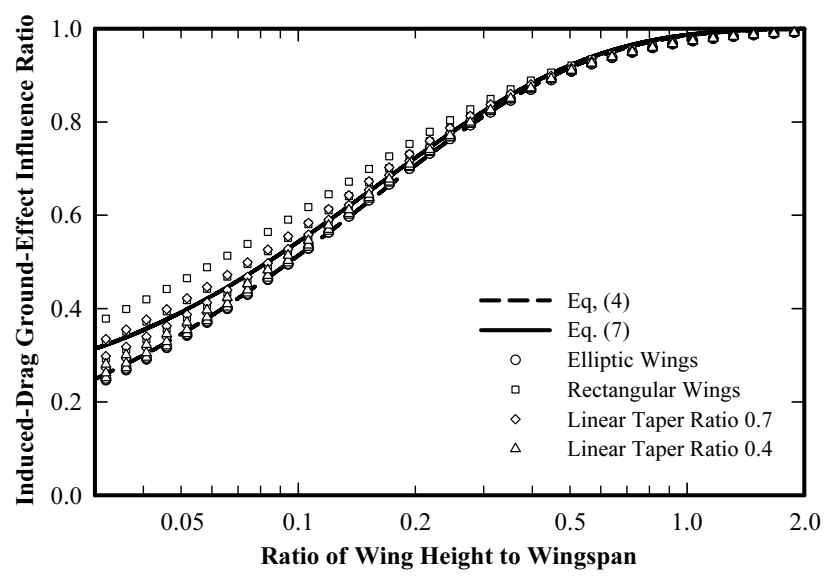

Fig. 4 Comparison of the small-angle lifting-line solutions and results obtained from Eq. (4) with results obtained from Eq. (7) using an aspect ratio of 6.0 and a lift coefficient of 0.5 .

It is widely acknowledge in the aeronautics literature that the induced-drag ground-effect influence ratio depends somewhat on the wing planform and aspect ratio as well as the dimensionless ratio, $h / b$. For example, following the development of McCormick [3], Suh and Ostowari [10] present a closed-form relation that describes this influence ratio as a function of $h / b$ and the well known Oswald wing efficiency, which depends on the wing planform. However, Laitone [11] points out that the relation presented by Suh and Ostowari [10] predicts negative induced drag in the limit as the wing approaches the ground. Laitone [11] suggests that this unrealistic behavior results in part from Suh and Ostowari's [10] assumption of a constant Oswald efficiency, when in fact the Oswald 
efficiency is also likely influenced by ground effect. Laitone [11] also points out the typographical error introduced by McCormick [3], which resulted in Eq. (2).

The closed-form approximations given by Eqs. (5) and (6) provide reasonable means for estimating the induced drag when the lift coefficient is known. This is typically the case for an airplane in free flight, where the angle of attack must take the value necessary to support the airplane's weight at a given airspeed. However, during ground roll, the angle of attack is commonly held constant by the landing gear, and because lift is also influenced by ground effect, the lift coefficient is not known a priori. Therefore, to estimate the lift and induced drag during ground roll, it would be useful to have a closed-form approximation for a lift ground-effect influence ratio, which is defined here to be the lift coefficient evaluated with the wing at some height $h$ above the ground divided by the lift coefficient at the same aerodynamic angle of attack, $\alpha$, evaluated with the wing outside the influence of ground effect,

$$
\text { lift ground-effect influence ratio } \equiv \frac{\left[C_{L}(\alpha)\right]_{h}}{\left[C_{L}(\alpha)\right]_{\infty}}
$$

Figure 5 shows values for this influence ratio as a function of $h / b$. These results were obtained from the same grid-resolved numerical lifting-line solutions that were used to obtain the induced-drag results shown in Figs. 2 through 4.

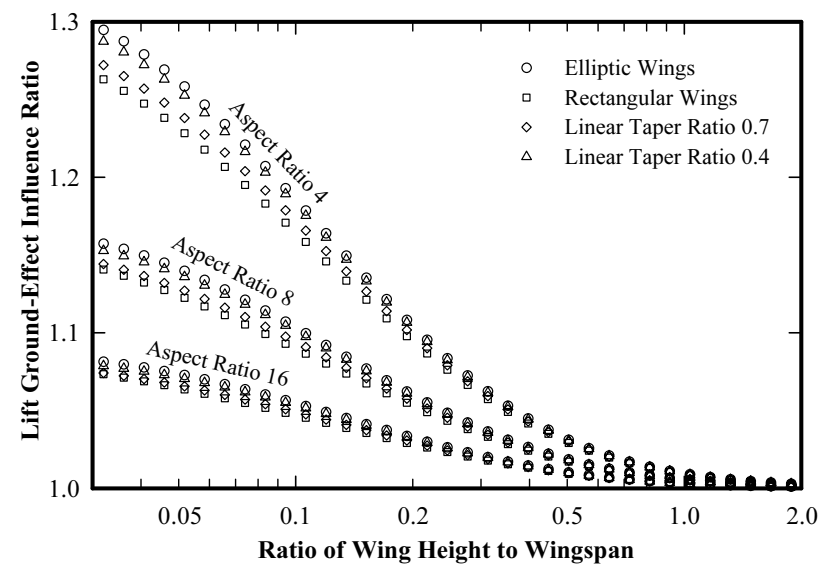

Fig. 5 Values for the lift ground-effect influence ratio obtained from the numerical lifting-line method of Phillips and Snyder [9] for aspect ratios of 4, 8, and 16 .

It can be seen from Fig. 5 that the influence of ground effect on the wing lift coefficient at constant angle of attack cannot be approximated reasonably as a unique function of the single dimensionless variable, $h / b$. The ratio 
of the lift coefficient in ground effect to that out of ground effect depends significantly on the wing aspect ratio and somewhat on the wing planform. Furthermore, for heights much less than the wingspan, ground effect can increase the lift coefficient by more than $20 \%$, and for these wings with no geometric or aerodynamic twist, the induced-drag coefficient is proportional to the lift coefficient squared. Hence, the influence of ground effect on the wing lift coefficient could increase the induced drag during ground roll by more than $40 \%$.

Over the past 5 decades considerable effort has been devoted to understanding and predicting the consequences of ground effect $[1,3,7,8,10-31]$. In the present work, closed-form relations for estimating induced-drag and lift coefficients for untwisted wings in ground effect are developed by correlating results obtained from numerical solutions [9] to Prandtl's lifting-line theory [32,33], which produces results in good agreement with inviscid CFD solutions [34] at a small fraction of the computational cost.

\section{Wings of Elliptic Planform}

The reduction in induced drag caused by ground effect is a direct result of a decrease in the downwash, which is induced on the wing by the vortex sheet that is shed from the wing. For untwisted wings of elliptic planform outside the influence of ground effect, lifting-line theory provides an analytic relation for the downwash induced by this shed vortex sheet. At small angles of attack, elliptic wings with no geometric or aerodynamic twist yield the downwash distribution given by the integral equation

$$
\begin{gathered}
V_{y}(\bar{x}, \bar{y}, \bar{z})=-\frac{V_{\infty} C_{L}}{\pi^{2} R_{A}} \int_{\phi=0}^{\pi}\left\{\frac{\cos (\phi)[\bar{z}+\cos (\phi)]}{\bar{y}^{2}+[\bar{z}+\cos (\phi)]^{2}}\right. \\
+\frac{\bar{x} \cos (\phi)[\bar{z}+\cos (\phi)]}{\left\{\bar{y}^{2}+[\bar{z}+\cos (\phi)]^{2}\right\}\left\{\bar{x}^{2}+\bar{y}^{2}+[\bar{z}+\cos (\phi)]^{2}\right\}^{1 / 2}} \\
\left.+\frac{\bar{x} \sin ^{2}(\phi)}{\left\{\bar{x}^{2}+\bar{y}^{2}+[\bar{z}+\cos (\phi)]^{2}\right\}^{3 / 2}}\right\} d \phi
\end{gathered}
$$

where $V_{y}$ is the $y$-component of induced velocity,

$$
\bar{x}=\frac{x}{b / 2}, \quad \bar{y}=\frac{y}{b / 2}, \quad \bar{z}=\frac{z}{b / 2}
$$

$x$ is the streamwise coordinate measured aft of the wing quarter chord, $y$ is the coordinate normal to both the freestream and the wingspan measured upward from the wing quarter chord, $z$ is the spanwise coordinate measured 
left from the wing midspan, $V_{\infty}$ is the freestream airspeed, and $R_{A}$ is the wing aspect ratio. The downwash induced along the wing quarter chord is obtained from Eq. (8) by setting both $x$ and $y$ to zero,

$$
\begin{aligned}
V_{y}(0,0, \bar{z}) & =-\frac{V_{\infty} C_{L}}{\pi^{2} R_{A}}\left\{\int_{\phi=0}^{\pi} \frac{\cos (\phi)}{[\bar{z}+\cos (\phi)]} d \phi\right\} \\
& =-\frac{V_{\infty} C_{L}}{\pi R_{A}}\left\{\begin{array}{ll}
\left(1+\frac{\bar{z}}{\sqrt{\bar{z}^{2}-1}}\right), & \bar{z}<-1 \\
\left(1-\frac{\bar{z}}{\sqrt{\bar{z}^{2}-1}}\right), & \bar{z}>1
\end{array}\right\}
\end{aligned}
$$

Hence, lifting-line theory predicts that an untwisted elliptic wing outside the influence of ground effect produces uniform downwash along the wing quarter chord.

Because the lifting-line solution for the downwash induced along the quarter chord of an untwisted elliptic wing is so simple, some insight into the nature of ground effect and the accuracy of the numerical solutions may be gleaned by comparing the downwash distribution predicted from Eq. (9) with that predicted from numerical solutions for the same wing at the same angle of attack, both in and outside of ground effect. Although a closedform solution for the downwash distribution on an elliptic wing in ground effect does not exist, the numerical lifting-line method of Phillips and Snyder [9] can be used to predict the downwash induced on a wing of any planform, both in and out of ground effect. The results shown in Fig. 6 were obtained from this numerical liftingline method using 400 horseshoe vortex elements across the wingspan of an untwisted elliptic wing of aspect ratio 6 outside the influence of ground effect. The results obtained from this numerical lifting-line solution agree with results predicted from Eq. (9) to four significant digits. Similar results are shown in Fig. 7 for the same wing at the same angle of attack but in ground effect with $h / b=0.1$. Notice that ground effect does not reduce the downwash uniformly across the wingspan. The reduction in downwash between the out-of-ground-effect solution and the inground-effect solution is slightly less than 10 percent at the wingtips and slightly more than 50 percent at the midspan. Hence, we see that an untwisted elliptic wing does not produce an elliptic lift distribution in ground effect. 


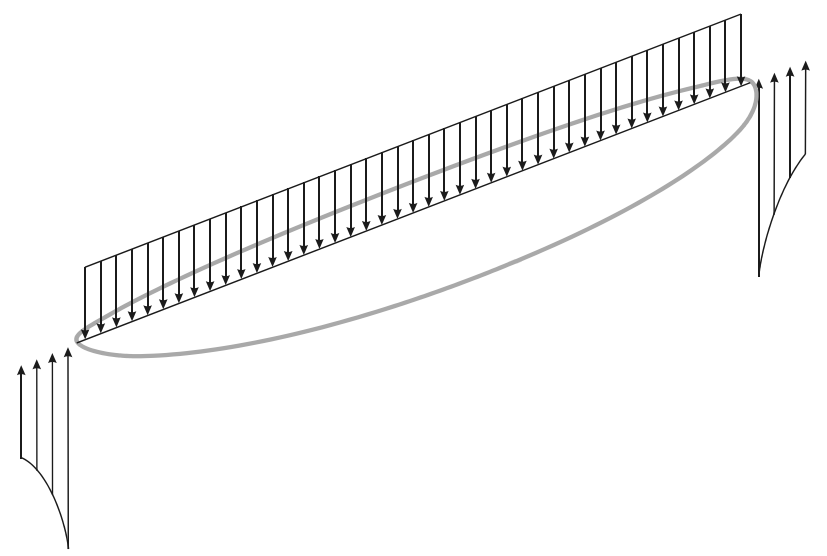

Fig. 6 Downwash along the quarter chord of an untwisted elliptic wing of aspect ratio 6, outside the influence of ground effect, as predicted from the numerical lifting-line method of Phillips and Snyder [9].

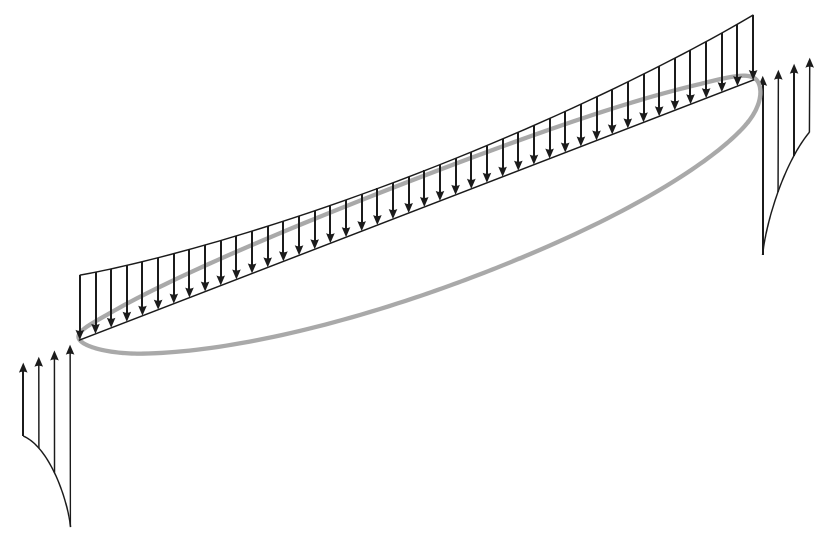

Fig. 7 Downwash along the quarter chord of an untwisted elliptic wing of aspect ratio 6, in ground effect with $h / b=0.1$, as predicted from the numerical lifting-line method of Phillips and Snyder [9].

Although the mathematics of lifting-line theory allow us to predict wing lift and induced-drag coefficients at very low values of $h / b$, the lowest values of $h / b$ shown in Figs. 2-5 are not of much practical interest. Because the wing quarter-chord line must remain sufficiently above the ground to permit air to flow freely around the airfoil sections of the wing, the absolute lower limit for wing height is fixed by the airfoil thickness and geometric angle of attack, not by the wingspan. Furthermore, lifting-line theory does not produce accurate results unless the wingspan is several times larger than the geometric mean chord length. Similarly, we should not expect lifting-line theory to produce accurate results unless the wing height is several times larger than the airfoil thickness. Typical wing aspect ratios are in the range of 6 to 8 and a typical airfoil thickness is about $12 \%$ of the chord length. Hence, assuming a 
minimum wing height of 5 times the airfoil thickness at the geometric mean chord, the practical lower limit on $h / b$ for the application of lifting-line theory to typical wings is in the range of about 0.075 to 0.1 .

Lift and induced-drag ground-effect influence ratios for untwisted elliptic wings at small aerodynamic angles of attack, as predicted from numerical lifting-line solutions for $h / b>0.07$, are shown in Fig. 8 for a wide range of aspect ratios. In this figure, separate curves are plotted for both of these influence ratios and 9 different aspect ratios ranging from 4 to 20 in steps of 2 . Notice that for the induced-drag ratio, all 9 curves are coincident to within the accuracy that could be expected from the numerical solutions. Hence, this induced-drag ratio for untwisted elliptic wing is, for all practical purposes, independent of aspect ratio.

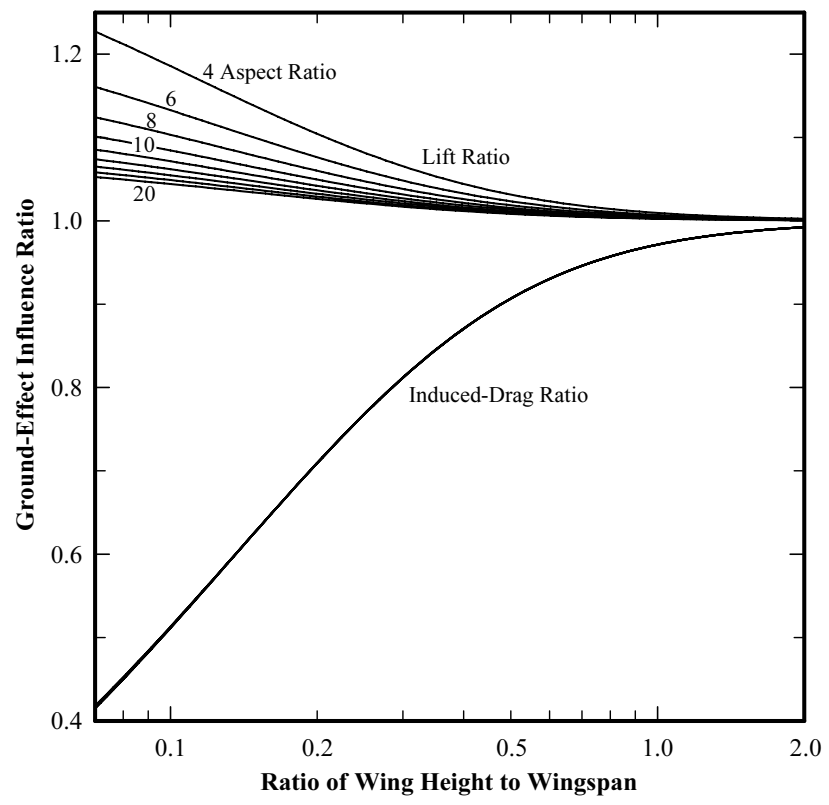

Fig. 8 Lift and induced-drag ground-effect influence ratios for untwisted elliptic wings of aspect ratios 4, 6, $8,10,12,14,16,18$, and 20, as obtained from the numerical lifting-line method of Phillips and Snyder [9].

The relation in Fig. 8 showing the induced-drag ground-effect influence ratio for untwisted elliptic wings at small aerodynamic angles of attack is very closely approximated by the closed-form relation

$$
\begin{aligned}
\frac{\left(C_{D i} / C_{L}^{2}\right)_{h}}{\left(C_{D i} / C_{L}^{2}\right)_{\infty}} & =1-\exp \left[-4.74(h / b)^{0.814}\right] \\
& -(h / b)^{2} \exp \left[-3.88(h / b)^{0.758}\right]
\end{aligned}
$$


Similarly, the lift ground-effect influence ratio for untwisted elliptic wings at small aerodynamic angles of attack is quite well approximated using the closed-form relation

$$
\frac{\left[C_{L}(\alpha)\right]_{h}}{\left[C_{L}(\alpha)\right]_{\infty}}=1+\frac{288(h / b)^{0.787} \exp \left[-9.14(h / b)^{0.327}\right]}{R_{A}^{0.882}}
$$

A comparison between results predicted from Eqs. (10) and (11) and results obtained from the numerical lifting-line solutions is shown in Fig. 9.

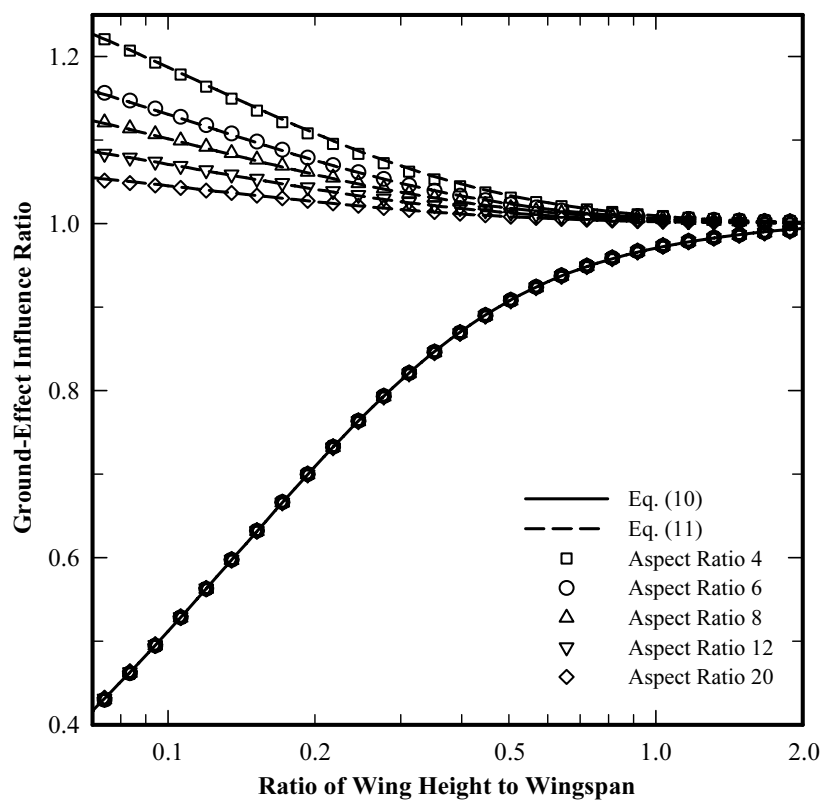

Fig. 9 Comparison of results obtained from Eqs. (10) and (11) with results obtained from the numerical lifting-line method of Phillips and Snyder [9] for wings of elliptic planform.

\section{Wings with Linear Taper}

As can be seen in Figs. 3 and 5, the ground-effect influence ratios for wings with linear taper do not precisely match those for wings of elliptic planform. Furthermore, these deviations depend on both the taper ratio and aspect ratio. For taper ratios near 0.3 , results obtained for wings with linear taper agree closely with those for wings of elliptic planform. However, for taper ratios near 1.0, the induced-drag ratio is somewhat higher than that for elliptic wings and the lift ratio is slightly lower than that for elliptic wings. 
Results obtained from numerical lifting-line solutions for the induced-drag ground-effect influence ratio for untwisted tapered wings at small aerodynamic angles of attack agree closely with the closed-form relation

$$
\begin{array}{r}
\frac{\left(C_{D i} / C_{L}^{2}\right)_{h}}{\left(C_{D i} / C_{L}^{2}\right)_{\infty}}=1-\delta_{D} \exp \left[-4.74(h / b)^{0.814}\right] \\
-(h / b)^{2} \exp \left[-3.88(h / b)^{0.758}\right] \\
\delta_{D}=1-0.157\left(R_{T}^{0.775}-0.373\right)\left(R_{A}^{0.417}-1.27\right)
\end{array}
$$

where $R_{T}$ is the wing taper ratio. Similarly, results obtained for the lift ground-effect influence ratio for untwisted tapered wings at small aerodynamic angles of attack are quite well approximated using the closed-form relation

$$
\begin{gathered}
\frac{\left[C_{L}(\alpha)\right]_{h}}{\left[C_{L}(\alpha)\right]_{\infty}}=1+\delta_{L} \frac{288(h / b)^{0.787} \exp \left[-9.14(h / b)^{0.327}\right]}{R_{A}^{0.882}} \\
\delta_{L}=1-2.25\left(R_{T}^{0.00273}-0.997\right)\left(R_{A}^{0.717}+13.6\right)
\end{gathered}
$$

Comparisons between results predicted from Eqs. (12)-(15) and results obtained from the numerical lifting-line solutions are shown in Figs. 10-12. The tapered-wing correction coefficients, $\delta_{D}$ and $\delta_{L}$, as given in Eqs. (13) and (15) were developed by correlating numerical lifting-line solutions for taper ratios between 0.3 and 1.0 and aspect ratios between 4 and 20. These closed-form approximations should be used with caution outside this range.

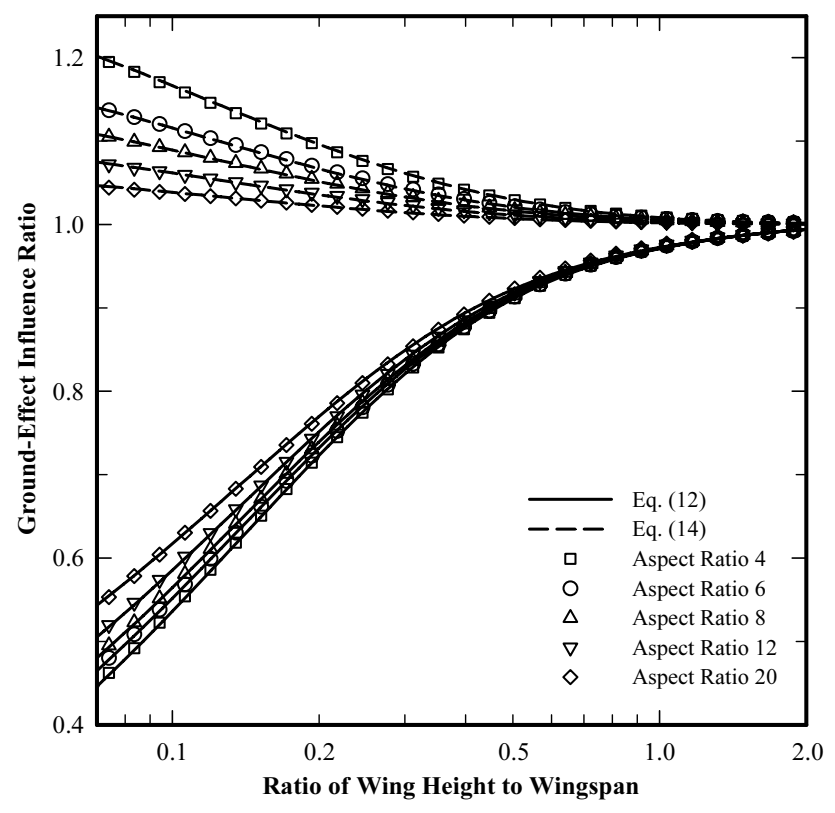

Fig. 10 Comparison of results obtained from Eqs. (12)-(15) with results obtained from the numerical liftingline method of Phillips and Snyder [9] for rectangular wings. 


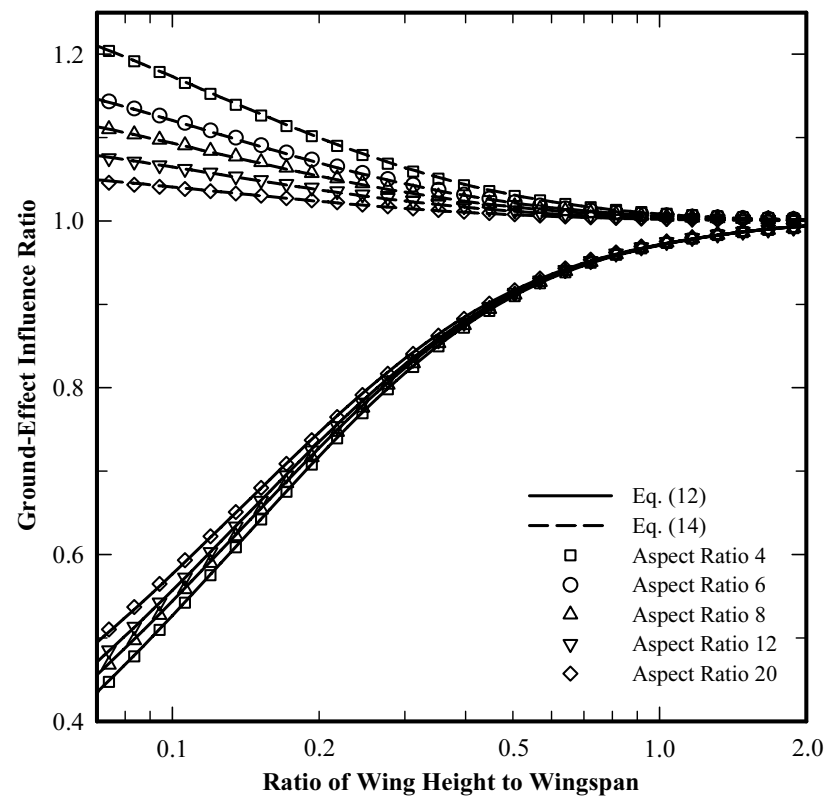

Fig. 11 Comparison of results obtained from Eqs. (12)-(15) with results obtained from the numerical liftingline method of Phillips and Snyder [9] for wings with a linear taper ratio of 0.7 .

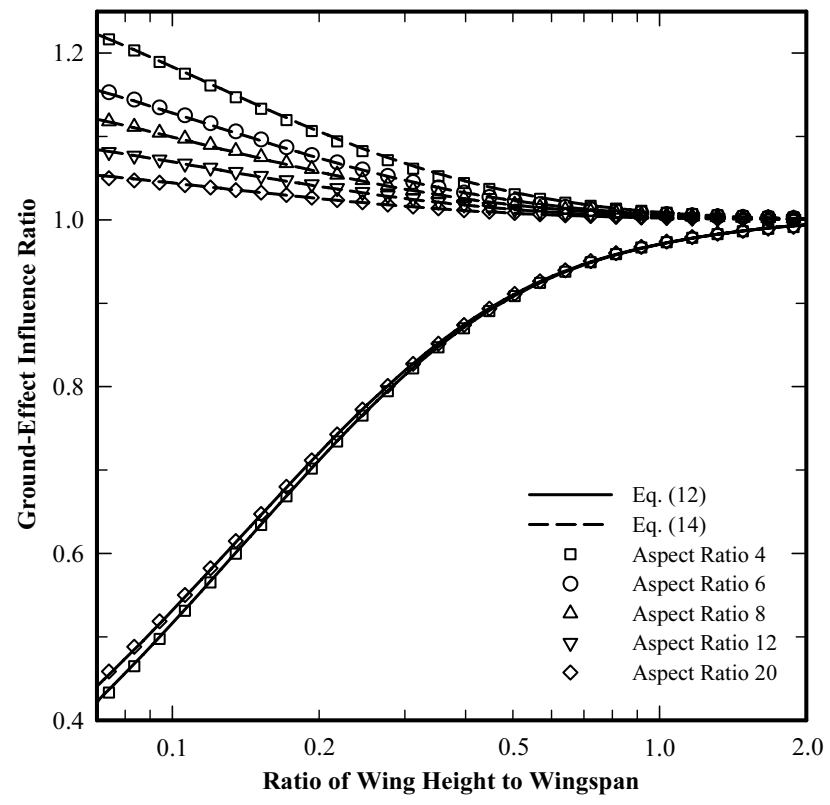

Fig. 12 Comparison of results obtained from Eqs. (12)-(15) with results obtained from the numerical liftingline method of Phillips and Snyder [9] for wings with a linear taper ratio of 0.4 . 


\section{Effects of High Lift Coefficient}

Strictly speaking, the ground-effect influence ratios predicted from Eqs. (10) and (11) or Eqs. (12)-(15) apply only to small aerodynamic angles of attack, i.e., small wing lift coefficients. However, the wing lift coefficient in ground effect is typically quite high. For free flight in ground effect, airspeeds typically range from about 1.1 to 1.2 times the stall speed, which usually requires wing lift coefficients near 1.0 or greater. For accelerating ground roll, the optimum lift coefficient is typically on the order of about 0.3 for a smooth paved runway, and can be as high 1.3 for a rough runway surface such as a grass strip. Hence, for best accuracy, results predicted from Eqs. (10) and (11) or Eqs. (12)-(15) should be corrected for a high lift coefficient.

At high lift coefficients, the induced-drag ratio is somewhat higher than that predicted by Eq. (10) or Eq. (12) and the lift ratio is slightly lower than that predicted by Eq. (11) or Eq. (14). Results obtained from numerical lifting-line solutions for the ground-effect influence ratios for untwisted wings at high aerodynamic angles of attack agree quite well with the closed-form relations

$$
\begin{aligned}
\frac{\left(C_{D i} / C_{L}^{2}\right)_{h}}{\left(C_{D i} / C_{L}^{2}\right)_{\infty}}= & \left\{1-\delta_{D} \exp \left[-4.74(h / b)^{0.814}\right]\right. \\
& \left.-(h / b)^{2} \exp \left[-3.88(h / b)^{0.758}\right]\right\} \beta_{D} \\
\beta_{D}= & 1+0.0361 C_{L}^{1.21} /\left[R_{A}^{1.19}(h / b)^{1.51}\right] \\
\frac{\left[C_{L}(\alpha)\right]_{h}}{\left[C_{L}(\alpha)\right]_{\infty}}= & \left\{1+\delta_{L} 288(h / b)^{0.787}\right. \\
\times & \left.\exp \left[-9.14(h / b)^{0.327}\right] / R_{A}^{0.882}\right\} / \beta_{L} \\
\beta_{L}= & 1+0.269 C_{L}^{1.45} /\left[R_{A}^{3.18}(h / b)^{1.12}\right]
\end{aligned}
$$

where $C_{L}$ is the lift coefficient in ground effect. The coefficients $\delta_{D}$ and $\delta_{L}$ are both 1.0 for elliptic wings and can be obtained from Eqs. (13) and (15), respectively, for wings with linear taper. Comparisons between results predicted from Eqs. (16)-(19) and results obtained from the numerical lifting-line solutions are shown in Figs. 13-16.

It is easily shown from Eqs. (17) and (19) that a high wing lift coefficient has a much greater effect on the induced-drag ground-effect influence ratio than it does on the lift ground-effect influence ratio. It should also be noted from Eqs. (17) and (19) that the effects of high lift coefficient on both ground-effect influence ratios decrease with increasing wing aspect ratio. For a lift coefficient of 1.2 or less and a typical wing height in ground roll with 
$h / b=0.1$ or greater, $\beta_{D}$ is less than 1.1 for any wing aspect ratio greater than 9.5 and $\beta_{L}$ is less than 1.01 for any wing aspect ratio greater than 6.9 .

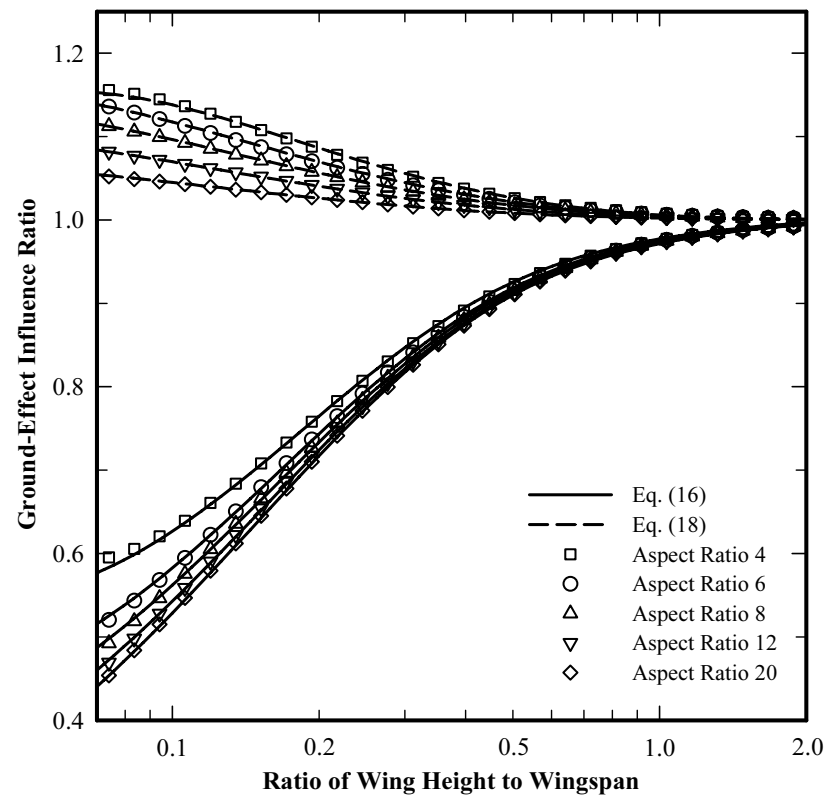

Fig. 13 Comparison of results obtained from Eqs. (16)-(19) with results obtained from the numerical liftingline method of Phillips and Snyder [9] for elliptic wings with $C_{L}=1.0$.

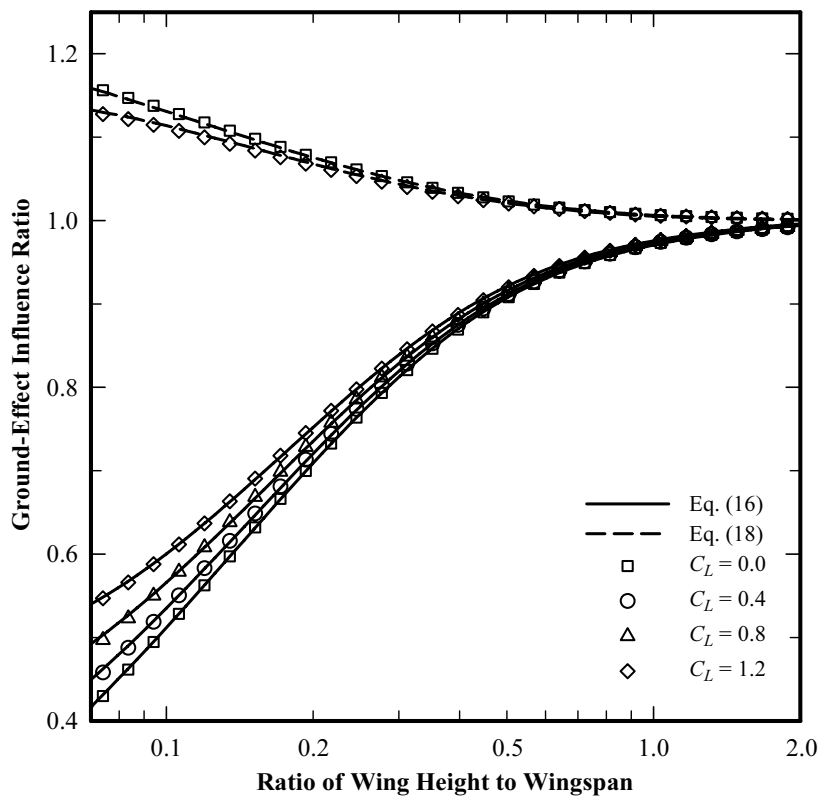

Fig. 14 Comparison of results obtained from Eqs. (16)-(19) with results obtained from the numerical liftingline method of Phillips and Snyder [9] for an elliptic wing of aspect ratio 6 at various lift coefficients. 


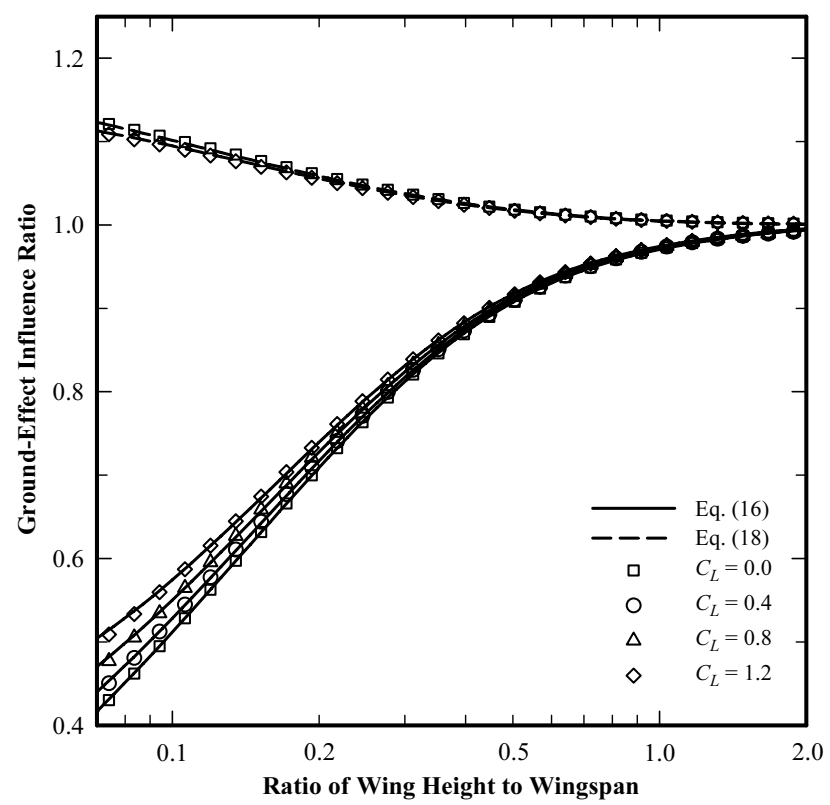

Fig. 15 Comparison of results obtained from Eqs. (16)-(19) with results obtained from the numerical liftingline method of Phillips and Snyder [9] for an elliptic wing of aspect ratio 8 at various lift coefficients.

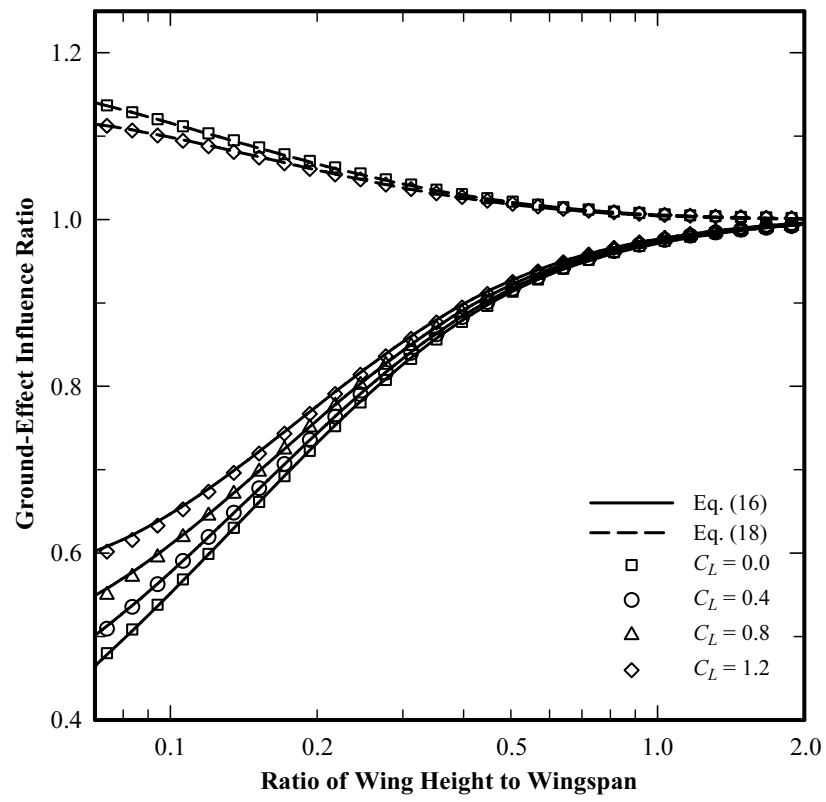

Fig. 16 Comparison of results obtained from Eqs. (16)-(19) with results obtained from the numerical liftingline method of Phillips and Snyder [9] for a rectangular wing of aspect ratio 6 at various lift coefficients.

\section{Conclusions}

For untwisted wings of elliptic planform operating at small lift coefficients with $h / b>0.07$, the closed-form relation attributed to Torenbeek [7] and repeated here in Eq. (4) agrees with the lifting-line results shown in Fig. 2 to 
within less than 1.5\%. However, when the lifting-line results shown in Fig. 2 for untwisted rectangular and tapered wings with $h / b>0.07$ are also considered, the maximum deviation from Eq. (4) is more than $19 \%$. A slightly improved closed-form approximation for the mean of all numerical lifting-line results shown in Fig. 2 was obtained in Eq. (5) by optimizing the coefficients in the relation of Torenbeek [7] to minimize the root-mean-square deviation from the lifting-line results. For small wing lift coefficients and $h / b>0.07$, Eq. (5) agrees with all lifting-line results shown in Fig. 2 for untwisted elliptic, rectangular, and tapered wings to within about 14\%.

During the early phases of airplane design, when the details of wing geometry are unknown, the closed-form approximation given by Eq. (5) provides a reasonable means for estimating the induced drag acting on a wing in ground effect. However, if the wing geometry is known, Eq. (16) provides better results. For $h / b>0.07$, results predicted from Eq. (16) agree with all lifting-line results shown in Figs. 2-4 and 8-16 for untwisted elliptic, rectangular, and tapered wings to within about $1.2 \%$. This closed-form approximation can be used to predict the induced-drag ground-effect influence ratio as a function of wing planform, aspect ratio, and lift coefficient, as well as the ratio of wing height to wingspan.

A method sometimes used during the early phases of design to estimate the influence of ground effect on induced drag is based on an approximation for the reduction in downwash due to ground effect at the wing midspan. This approximate midspan reduction in downwash is assumed to be constant across the entire span of the wing and the reduction in induced drag is computed accordingly. Results presented here in Figs. 6 and 7 show that the reduction in downwash due to ground effect at the wing midspan can be several times larger than that at the wingtips. Furthermore, because much of the induced drag acting on a wing is generated near the wingtips, assuming the midspan reduction in downwash to be constant across the entire wingspan will substantially under predict the induced drag in ground effect.

To evaluate the induced-drag coefficient in ground effect from knowledge of the induced-drag ground-effect influence ratio, the wing lift coefficient must be known. For an airplane in free flight, the wing lift coefficient can be determined from the airplane's weight and airspeed. However, when the angle of attack is held constant and a portion of the airplane's weight is supported by the landing gear during ground roll, the influence of ground effect on the wing lift coefficient at constant angle of attack must be considered. Equation (18) provides an accurate closed-form approximation that can be used for this purpose. Results presented here show that the lift ground-effect 
influence ratio predicted by Eq. (18) is a strong function of the wing aspect ratio and the ratio of wing height to wingspan, but only a weak function of the wing planform and lift coefficient.

All of the closed-form approximations given by Eqs. (10)-(19) were developed by correlating numerical lifting-line results for elliptic, rectangular, and tapered wings having no sweep, dihedral, or twist. Wing aspect ratios were limited to the range from 4 to 20 and wing taper ratios were limited to the range from 0.3 to 1.0 . Only wing heights greater than 0.07 times the wingspan and wing lift coefficients of 1.2 or less were included in these correlations. The closed-form approximations presented here should be used with caution outside the range of parameters for which they were developed.

\section{References}

[1] Hoerner, S., and Borst, H., "Lift of Airplane Configurations," Fluid Dynamic Lift, Hoerner Fluid Dynamics, Bricktown, NJ, 1975, pp. 20-1-20-22.

[2] Raymer, D. P., "Ground Effect," Aircraft Design: A Conceptual Approach, 5th ed., American Institute of Aeronautics and Astronautics, Washington, D.C., 2012, p. 463.

[3] McCormick, B. W., "Ground Roll," Aerodynamics, Aeronautics, and Flight Mechanics, 1st ed., Wiley, New York, 1979, pp. 419-424.

[4] Anderson, J. D., "Calculation of Ground Roll," Aircraft Performance and Design, McGraw-Hill, New York, 1999, pp. 355-363.

[5] Anderson, J. D., “Takeoff Performance,” Introduction to Flight, 7th ed., McGraw-Hill, New York, 2012, pp. 520-526.

[6] Phillips, W. F., "Takeoff and Landing Performance," Mechanics of Flight, 2nd ed., Wiley, Hoboken, NJ, 2010, pp. 337-353.

[7] Torenbeek, E., "Ground Effects," Synthesis of Subsonic Airplane Design, Delft University Press, Delft, The Netherlands, 1982, pp. 551-554.

[8] McCormick, B. W., "Ground Roll," Aerodynamics, Aeronautics, and Flight Mechanics, 2nd ed., Wiley, New York, 1995, pp. 358-365.

[9] Phillips, W. F., and Snyder, D. O., “Modern Adaptation of Prandtl's Classic Lifting-Line Theory,” Journal of Aircraft, Vol. 37, No. 4, 2000, pp. 662-670. 
[10] Suh, Y. B., and Ostowari, C., "Drag Reduction Factor Due to Ground Effect," Journal of Aircraft, Vol. 25, No. 11, 1988, pp. 1071-1072.

[11] Laitone, E. V., "Comment on 'Drag Reduction Factor Due to Ground Effect'," Journal of Aircraft, Vol. 27, No. 1, 1990, pp. 96-96.

[12] Schweikhard, W., "A Method for In-Flight Measurement of Ground Effect on Fixed-Wing Aircraft," Journal of Aircraft, Vol. 4, No. 2, 1967, pp. 101-104.

[13] Sachs, G., "Change in Pitching-Moment Coefficient Due to Ground Effect," Journal of Aircraft, Vol. 6, No. 6, 1969, pp. 573-574.

[14] Davis, J. E., and Harris, G. L., "Nonplanar Wings in Nonplanar Ground Effect," Journal of Aircraft, Vol. 10, No. 5, 1973, pp. 308-312.

[15] Mamada, H., and Ando, S., "Minimum Induced Drag of a Semi-Circular Ground Effect Wing," Journal of Aircraft, Vol. 10, No. 11, 1973, pp. 660-663.

[16] Ailor, W. H., and Eberle, W. R., "Configuration Effects on the Lift of a Body in Close Ground Proximity," Journal of Aircraft, Vol. 13, No. 8, 1976, pp. 584-589.

[17] Staufenbiel, R., "Some Nonlinear Effects in Stability and Control of Wing-in-Ground Effect Vehicles," Journal of Aircraft, Vol. 15, No. 8, 1978, pp. 541-544.

[18] Lange, R. H., and Moore, J. W., "Large Wing-in-Ground Effect Transport Aircraft," Journal of Aircraft, Vol. 17, No. 4, 1980, pp. 260-266.

[19] Er-El, J., and Weihs, D., "Ground Effect on Slender Wings at Moderate and High Angles of Attack," Journal of Aircraft, Vol. 23, No. 5, 1986, pp. 357-358.

[20] Plotkin, A., and Tan, C. H., "Lifting-line Solution for a Symmetrical Thin Wing in Ground Effect," AIAA Journal, Vol. 24, No. 7, 1986, pp. 1193-1194.

[21] Plotkin, A., and Dodbele, S., "Slender Wing in Ground Effect," AIAA Journal, Vol. 26, No. 4, 1988, pp. 493-494.

[22] Lee, P., Lan, C. E., and Muirhead, V. U., "Experimental Investigation of Dynamic Ground Effect,” Journal of Aircraft, Vol. 26, No. 6, 1989, pp. 497-498.

[23] Mook, D. T., and Nuhait, A. O., "Numerical Simulation of Wings in Steady and Unsteady Ground Effects," Journal of Aircraft, Vol. 26, No. 12, 1989, pp. 1081-1089. 
[24] Chawla, M. D., Edwards, L. C., and Franke, M. E., "Wind-Tunnel Investigation of Wing-in-Ground Effects," Journal of Aircraft, Vol. 27, No. 4, 1990, pp. 289-293.

[25] Liu, H., Hwang, P. A., and Srnsky, R. A., "Physical Modeling of Ground Effects on Vortex Wakes," Journal of Aircraft, Vol. 29, No. 6, 1992, pp. 1027-1034.

[26] Zerihan, J., and Zhang, X., "Aerodynamics of a Single Element Wing in Ground Effect," Journal of Aircraft, Vol. 37, No. 6, 2000, pp. 1058-1064.

[27] Zhang, X., and Zerihan, J., "Aerodynamics of a Double-Element Wing in Ground Effect," AIAA Journal, Vol. 41, No. 6, 2003, pp. 1007-1016.

[28] King, R. M., and Gopalarathnam, A., "Ideal Aerodynamics of Ground Effect and Formation Flight," Journal of Aircraft, Vol. 42, No. 5, 2005, pp. 1188-1199.

[29] Lee, J., Han, C., and Bae, C., "Influence of Wing Configurations on Aerodynamic Characteristics of Wings in Ground Effect,” Journal of Aircraft, Vol. 47, No. 3, 2010, pp. 1030-1040.

[30] Molina, J., and Zhang, X., “Aerodynamics of a Heaving Airfoil in Ground Effect," AIAA Journal, Vol. 49, No. 6, 2011, pp. 1168-1179.

[31] Lee, S., and Lee, J., "Optimization of Three-Dimensional Wings in Ground Effect Using Multiobjective Genetic Algorithm,” Journal of Aircraft, Vol. 48, No. 5, 2011, pp. 1633-1645.

[32] Prandtl, L., "Tragflügel Theorie," Nachricten von der Gesellschaft der Wissenschaften zu Güttingen, Geschäeftliche Mitteilungen, Klasse, 1918, pp. 451-477.

[33] Prandtl, L., “Applications of Modern Hydrodynamics to Aeronautics,” NACA TR-116, June 1921.

[34] Phillips, W. F., Fugal, S. R., and Spall, R. E., "Minimizing Induced Drag with Wing Twist, ComputationalFluid-Dynamics Validation,” Journal of Aircraft, Vol. 43, No. 2, 2006, pp. 437-444. 